\title{
A Multi-outputs DC Power Module
}

\author{
Fengchen $\mathrm{Li}^{1, \mathrm{a}}$, Dongguang $\mathrm{Zuo}^{1,}$, , Longtao $\mathrm{Hu}^{1, \mathrm{~b}}$ \\ ${ }^{1} X i$ 'an Research Institute of High Technology, Xi 'an 710025, China; \\ a610580194@qq.com, ${ }^{\mathrm{b}} 523098802 @ q q . c o m$
}

Keywords: DC/DC, isolation, voltage regulator, multi-output

\begin{abstract}
In order to meet the requirements which a variety of DC power supply of a certain test equipment, a voltage stabilizing module which can provide a variety of DC voltage is designed. The voltage stabilizing module comprises a power supply switch, a DC/DC switch power supply, a $\mathrm{DC} / \mathrm{DC}$ isolation module, a voltage stabilizing module and so on. The working principle and the realization method of the circuits are introduced in this paper. The power supply module has the advantages of stable output voltage and small static current.
\end{abstract}

\section{Introduction}

With the increasing development of electronic technology, power technology is used in the field of computer, industrial instrumentation, military and so on. Related to all walks of our life. The electronic equipments, which requires the power supply module to provide a variety of different voltage signals, have more and more functions.

In the development of a simulation test equipment. To meet the requirements of the equipment, a variety of DC voltage signal should be provided. Through the analysis, the precision of the test equipment is higher, and the space of the power supply module is small. To solve the above problems. A multi output DC voltage stabilized power supply module is designed, which is used to provide multiple $+5 \mathrm{~V}$ and $+3 \mathrm{~V}$ stable $\mathrm{DC}$ voltage signals to the equipment. The power supply has the advantages of low output noise, small static current and so on. The power supply satisfies the requirement of the voltage of the equipment.

\section{Overall Design}

The power module of the system structure diagram is shown in Figure 1. As we can see, the power switch converts the $220 \mathrm{~V} 50 \mathrm{~Hz}$ alternating current to the $24 \mathrm{~V}$ DC voltage signal. $24 \mathrm{~V} \mathrm{DC}$ voltage signal is divided into two signals by the DC/DC switch power supply. The two signals go through DC/DC isolation module and voltage regulator modules respectively. Multi-channel $5 \mathrm{~V}$, $3 \mathrm{~V}$ voltage regulator $\mathrm{DC}$ signal is obtained.

The DC/DC switching power supply will convert $24 \mathrm{~V}$ DC to $5 \mathrm{~V}$ working power supply. The switching power supply is a new production of small power switch module, using micro electronics technology, the small surface mounted integrated circuit and microelectronic components are assembled into a whole to form. A wide input voltage range, small output voltage ripple and high conversion efficiency can be provided. The DC/DC isolation module uses a transformer to isolate the input voltage and the output voltage, which can eliminate the ground loop and avoid the distortion in the signal transmission process. Voltage regulator module is mainly stable voltage, which provides $3 \mathrm{~V}$ and $5 \mathrm{~V}$ stable voltage signals for the whole equipment.

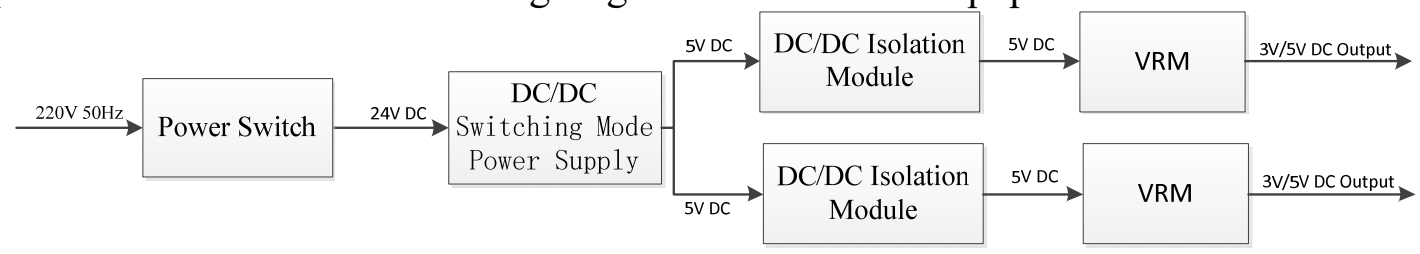

Fig. 1 System overall composition 


\section{Hardware Design}

\subsection{DC/DC Switching Power Supply}

The core part of the switch power supply voltage regulator is $1 \mathrm{~m} 2596$ Switching voltage regulator. The LM2596 series of regulators are monolithic integrated circuits that provide all the active functions for a step-down (buck) Switching regulator, capable of driving a 3A load with excellent line and load regulation. Requiring a minimum number of external components, these regulators are simple to use and include internal frequency compensation, and a fixed-frequency oscillator. The LM2596 series operates at a switching frequency of $150 \mathrm{kHz}$ thus allowing smaller sized filter components than what would be needed with lower frequency switching regulators. Other features include an ensured $\pm 4 \%$ tolerance on output voltage under specified input voltage and output load conditions, and $\pm 15 \%$ on the oscillator frequency. External shutdown is included, featuring typically $80 \mu \mathrm{A}$ standby current. Self protection features include a two stage frequency reducing current limit for the output switch and an over temperature shutdown for complete protection under fault conditions.

The design of external elements is mainly for anti interference, the input capacitance $\mathrm{C} 1-\mathrm{a}$ low ESR aluminum or tantalum bypass capacitor is needed between the input pin and ground pin. It must be located near the regulator using short leads. This capacitor prevents large voltage transients from appearing at the input, and provides the instantaneous current needed each time the switch turns on.

An output capacitor $\mathrm{C} 2$ is required to filter the output and provide regulator loop stability. Low impedance or low ESR Electrolytic or solid tantalum capacitors designed for switching regulator applications must be used.

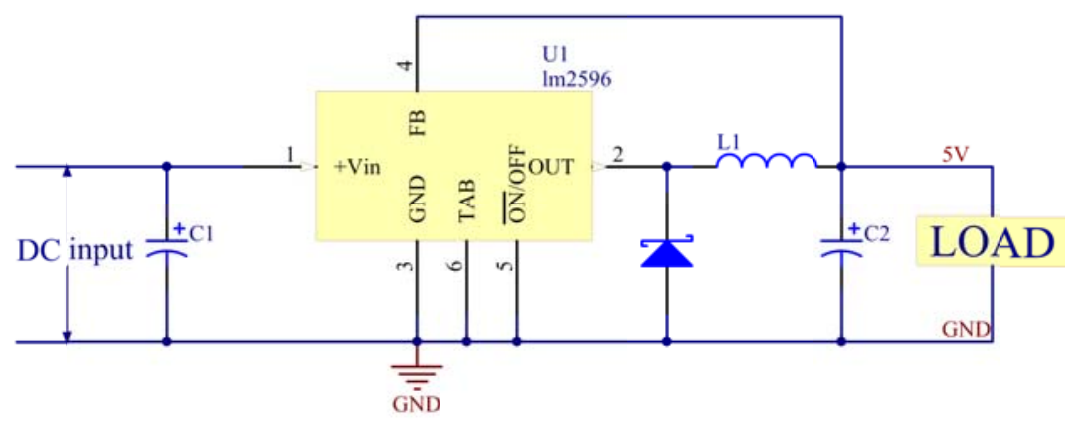

Fig. 2 DC/DC switching power supply

\section{2 $\mathrm{DC} / \mathrm{DC}$ isolation module}

To eliminate ground loops and ensure the power supply module, stable and reliable operation, so this DC/DC isolated module is added. The isolation module is a kind of DC/DC switching power supply, but the output and input voltages are not altogether, insulated by transformer. The switching power supply provides 5V DC. The voltage signal goes through DCP020507. The DCP02 series is a family of unregulated, isolated DC/DC converters. Requiring a minimum of external components and including on-chip device protection, the DCP02 series provides extra features such as synchronization of switching frequencies. It has conversion efficiency up to $89 \%$.

Multiple DC regulated power supply is needed to provide. Therefore, two DCP020507 is necessary. In order to guarantee the synchronization of the switching frequency, the 28 pin of DCP020507 is connected (as shown in Figure 3). In order to reduce the fluctuation and steady the voltage, $10 \mathrm{uF}$ tantalum capacitors should be used on the Vin pin and Vout pin.
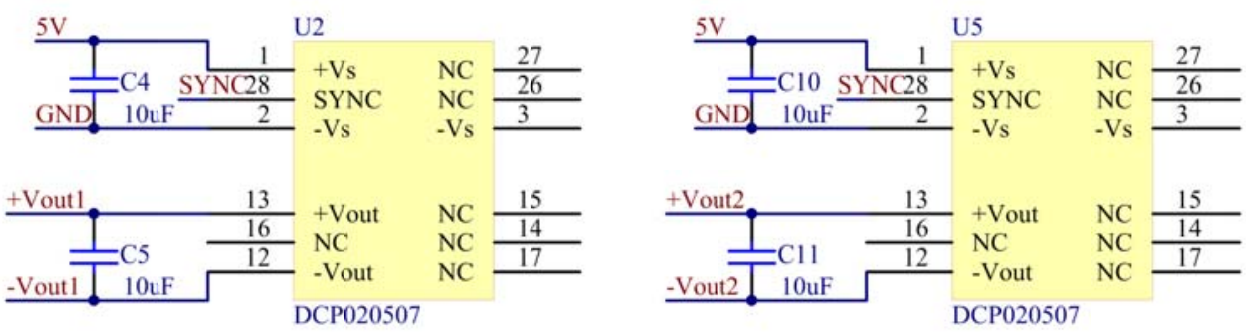

Fig. 3 DC/DC isolation module 


\subsection{Regulator modular}

The power module is mainly to provide $3.3 \mathrm{~V}, 5 \mathrm{~V}$ DC regulated signal, so the LDO is mainly used in this module. Relatived to the traditional regulator, LDO is a low voltage linear regulator. LDO is a new generation of integrated regulator, which is different from the three terminal regulators where LDO is a very low self consumption of the micro chip system. The structure of LDO includes starting circuit, constant current source bias unit, enable circuit, adjusting element, reference source, error amplifier, feedback resistor network and protection circuit and so on. It has the advantages of lower output noise, smaller static current, and fewer external components.

As shown in Figure 4, regulator module and the specific circuit, the main use of LM1117-5 lm1117-3.3 two fixed output types, the Vout pin of LM1117-5 is connected with load circuit and the Vin pin of LM1117-3.3 at the same time. In order to improve the transient response and stability, $10 \mathrm{uF}$ tantalum capacitors are used in the Vin pin and Vout pin.
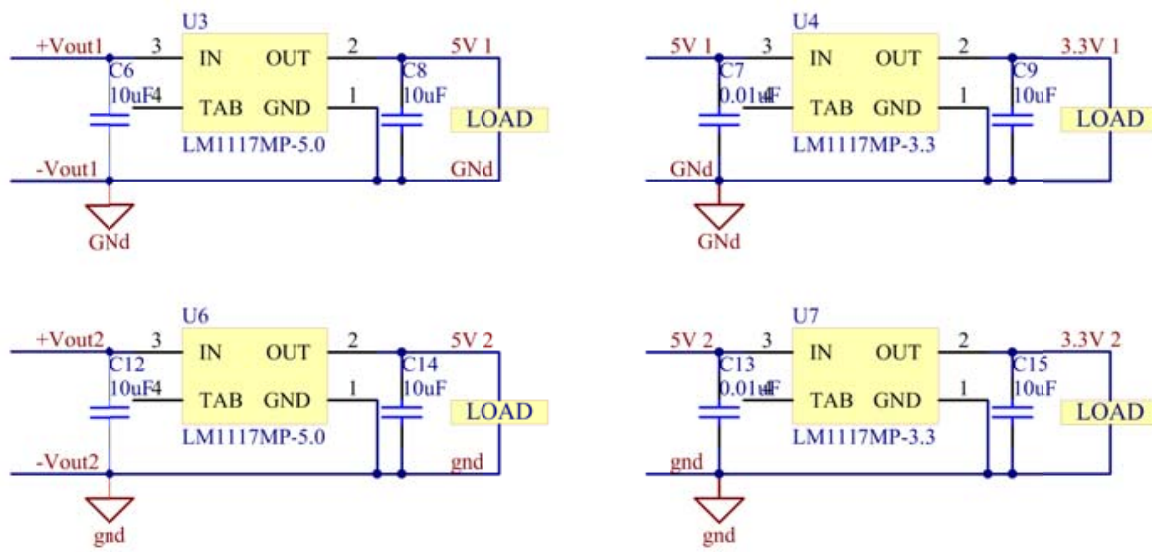

Fig. 4 Regulator modular

\section{Summary}

In the thesis, we design a multi output DC regulated power supply module. Each part of the circuit and chips are introduced in detail in this paper. Anti-interference is designed in the circuit, it has the advantages of low output noise, small quiescent current and etc. The requirements of the device are met.

\section{Reference}

[1]. Shutuan Zhang, Junxian Shi, Zhicai Xiao. Design and Implement of Multi-output DC Power Module [J]. Marine Electric \& Electronic Technology, Vol.31 (2011) No. 11, p.62-64

[2]. Zhanyou Sha, Yong Liu, Bingju An. Circuit Design of AC/DC Switch Power Module [J]. Electrical Measurement \& Instrumentation, (1999) No. 9, p.22-41

[3]. Jianbing Li, Kai Xie, Renjie Cheng. Design on Small Mine Intrinsic Safe DC-DC Isolated Power Modular [J]. Coal Science and Technology, Vol.39 (2011) No.6, p.75-77

[4]. Huilan Gou, Guangcao Liu. Minimum System and Serial Communication Implementation Based on STM32 [J] Industrial Control Computer, Vol.25 (2012) No.9, p26-28 